\title{
The Isolation and Properties of Non-restricting Mutants of Two Different Host Specificities Associated with Drug Resistance Factors
}

\author{
By DIANE BANNISTER* AND S. W. GLOVER \\ $M R C$ Molecular Genetics Unit, Department of Molecular Biology, \\ University of Edinburgh, Mayfield Road, Edinburgh, EH9 3 JR
}

\section{(Accepted for publication ro December 1969)}

\begin{abstract}
Non-restricting $\left(\mathrm{r}^{-}\right)$mutants of two different host specificities carried by resistance transfer factors have been isolated. As previously found with other host specificities, the non-restricting mutants were of two phenotypes: those that retained the ability to modify DNA, and those which had lost the ability to modify DNA. These mutants were tested for complementation with wild-type host specificities carried either on the Escherichia coli chromosome, on resistance transfer factors, or on the phage PI. No complementation was observed and possible explanations for this finding are considered.
\end{abstract}

\section{INTRODUCTION}

The host range of some bacteriophages can be altered as a result of a single cycle of growth in a new host strain, and this alteration is termed 'host controlled modification' (Luria, 1953; Arber \& Dussoix, 1962). In Escherichia coli strains K I2 and $\mathrm{B}$, afterwards called $\mathrm{K}$ and $\mathrm{B}$, two distinct host specificity functions, restriction and modification, have been recognized which operate with the same specificity. Restriction involves cleavage of at least one strand of the non-modified phage DNA (Meselson \& Yuan, 1968). Modification is specifically associated with the phage DNA also, and protects the phage from this restriction. In the case of the male specific phage fd, modification involves methylation of adenine residues in the DNA (Arber, I968).

The chromosomal genes determining these two phenotypic characters have been mapped close to thr and serB in Escherichia coli, both by bacterial conjugation and by co-transduction with these markers by phage $\mathrm{P}_{\mathrm{I}}$, and the strain specificities $\mathrm{K}$, B and I5 were shown to be allelic (Boyer, 1964; Colson, Glover, Symonds \& Stacey, 1965; Hoekstra \& De Haan, 1965; Lederberg, I966; Wood, 1966; Glover \& Colson, 1969). However, the elucidation of the genetic fine structure of the host specificity genes has not been feasible, mainly due to the lack of quantitative techniques for selecting clones of different restriction or modification phenotypes. Information concerning the number and function of these genes has come from study of the phenotypes of non-restricting mutants. The non-restricting $\left(\mathrm{r}^{-}\right)$mutants found have been of two phenotypes, those retaining modification ability $\left(\mathrm{m}^{+}\right)$, and those which were also defective in modification $\left(\mathrm{m}^{-}\right)$(Colson et al. I965; Wood, I965, 1966; Lederberg,

\footnotetext{
* Present address: Nuffield Unit of Medical Genetics, Department of Medicine, Crown Street, Liverpool, L69 3BX.
} 
I966). The existence of two phenotypes for non-restricting mutants suggested that the genetic determinants for host specificity involved a minimum of two genes. Conformation of this hypothesis has been obtained as a result of complementation studies in partial diploids constructed with $\mathrm{F}$ prime factors. These studies have revealed that at least two genes are involved in restriction; that one of these genes, together with a third, is required for modification; and a fourth gene has been postulated (Boyer \& Roulland-Dussoix, 1969; Glover, unpublished results).

Two host specificities, hsI and hsII (Bannister \& Glover, I968), are associated with the drug-resistance factors ( $\mathrm{R}$ factors) responsible for the transmissible resistance to antibiotics in Enterobacteriaceae (Watanabe, 1963; Meynell, Meynell \& Datta, 1968). In this paper we present a genetic analysis of the determinants involved in the control of these host specificities, based upon exploration of the types of restrictionless mutants which can be obtained, and the behaviour of these mutants in complementation tests.

\section{METHODS}

Media. Oxoid no. 2 broth: Oxoid no. 2 broth powder 25 g.; distilled water I.० 1. Oxoid agar: Oxoid no. 2 broth solidified with $\mathrm{I} 2.5$ g./l. Davis New Zealand agar. VB agar: VB salts (Vogel \& Bonner, I956) solidified with I5 g./l. Davis New Zealand agar. Water top agar: Difco Bacto agar 6 g.; distilled water $\mathrm{I} \cdot 01$. Difco agar: Oxoid tryptone Io g.; $\mathrm{NaCl} 8$ g.; glucose I g.; Difco Bacto agar Io g.; distilled water I.o 1. Sugars used at a final concentration of $2 \%(\mathrm{w} / \mathrm{v})$. Tetracycline used at a final concentration of $50 \mu \mathrm{g} . / \mathrm{ml}$. Streptomycin used at $20 \mu \mathrm{g}$. $/ \mathrm{ml}$. Amino acids used at $20 \mu \mathrm{g} . / \mathrm{ml}$.

Bacteriophages: phage $\lambda$ (Lederberg \& Lederberg, 1953) and its virulent mutant, $\lambda$ vir (Jacob \& Wollman, I954).

Bacterial strains. The strains of Escherichia coli used are listed in Table I.

Table I. Bacterial strains

\begin{tabular}{|c|c|c|}
\hline Strain & Characteristics & Reference \\
\hline c60o & thr leu thi lac hsK & Appleyard, I954 \\
\hline C600 (PI) & PI lysogen of c 600 & $\begin{array}{l}\text { Glover, Schell, Symonds } \\
\text { \& Stacey, I } 963\end{array}$ \\
\hline $\mathrm{c} 600(\lambda \mathrm{cr} 857)$ & $\lambda \mathrm{Cr} 857$ lysogen of $\mathrm{c} 600$ & Sussman \& Jacob, 1962 \\
\hline J $5-3$ & pro met $h s K(\lambda)$ & Clowes \& Rowley, I954 \\
\hline $4 \mathrm{~K}$ & thr leu thi serB lac $\mathrm{r}^{-} \mathrm{m}^{-} \mathrm{K}$ & Colson et al. 1965 \\
\hline $7 \mathrm{~K}$ & thr leu thi $\mathrm{r}^{-} \mathrm{m}^{+} \mathrm{K}$ & Colson et al. 1965 \\
\hline 15 & thi thy hsI 5 & Stacey, 1965 \\
\hline $\mathrm{C}$ & $t h r$ & Glover \& Colson, 1969 \\
\hline B & $\operatorname{trp} h s B$ & Glover \& Colson, 1969 \\
\hline B 6 & thr leu ilvA $\mathrm{r}^{-} \mathrm{m}^{+} \mathrm{B}$ & Glover \& Colson, 1969 \\
\hline B 8 & thr leu ilvA $\mathrm{r}^{-} \mathrm{m}-\mathrm{B}$ & Glover \& Colson, 1969 \\
\hline
\end{tabular}

$R$ factors. R I24 $f^{+}$and carrying resistance to tetracycline; R I32 $f^{-}$and carrying resistance to streptomycin and sulphonamide; $\mathrm{R} I 99 \mathrm{fi}^{-}$and carrying resistance to tetracycline; R 3I3-T-I $f^{-}$and carrying resistance to tetracycline. R I 24 carries hsI, the other three $\mathrm{R}$ factors carry hsII (hsI and hsII define the host specificities determined by these $\mathrm{R}$ factors (Bannister, 1969)). The $\mathrm{R}$ factors were the gift of $\mathrm{Dr}$ Elinor Meynell and Dr Naomi Datta. 


\section{Techniques}

Overnight cultures of bacteria were obtained by inoculating $5 \mathrm{ml}$. of Oxoid no. 2 broth from a single colony and incubating at $37^{\circ}$. Efficiency of plating (e.o.p.) is the ratio of the plaque count on the test strain to the plaque count on a permissive strain. A quick test for restriction was employed in the isolation of restrictionless mutants. Colonies were streaked on Difco agar plates and spotted with approximately $0.0 \mathrm{I}$ ml. of dilutions of phage $\lambda$ vir.

\section{Transfer of $R$ factors}

An overnight culture of the donor strain was diluted $\mathrm{r} / \mathrm{ro}$ into fresh broth, and incubated on an inclined rotor at $37^{\circ}$ for $\mathrm{I} \frac{1}{2} \mathrm{hr}$. This culture was diluted I/IO into an overnight culture of recipient and incubated at $37^{\circ}$ for $30 \mathrm{~min}$., then streaked out on VB agar selective for $\mathrm{R}^{+}$clones of the recipient, and incubated at $37^{\circ}$ for 24 to $36 \mathrm{hr}$. Colonies were purified by two serial single-colony isolations, and tested for all the characters of the recipient and the $\mathrm{R}$ factor.

\section{RESULTS}

\section{Spontaneous restrictionless mutants of hsII}

Routinely, $\mathrm{R}^{+}$strains were grown overnight from inocula taken from single colonies only. On two separate occasions, the $\mathrm{R}^{+}$colony used as an inoculum was found to be a non-restricting mutant, retaining modification ability (i.e. $\mathrm{r}^{-} \mathrm{m}^{+}$). These mutants were obtained from two different R factors, R I99 and R3I3-T-I, and the two mutant $\mathrm{R}$ factors were termed R I99-I and R3I3-I respectively.

\section{Restrictionless mutants produced by ethylmethane sulphonate mutagenesis}

Although two spontaneous mutants of the hsII host specificity had arisen, screening several hundred single colonies of strain J5-3 carrying R3I3-T-I hsII, and of J 5-3 R I 24 hsI, failed to reveal any other restrictionless mutants. To obtain additional mutants of both hsI and hsII, mutagenesis with ethylmethane sulphonate was used, and the mutagenesis was followed by an enrichment procedure developed by Hubachek \& Glover (in preparation). This procedure relies on the fact that $\lambda$.K can lysogenize restrictionless mutants of hsI or hsII efficiently and cannot readily lysogenize wild-type restricting bacteria. The mutagenesis was performed in $\mathrm{R}^{+}$strains lysogenic for the heat-inducible phage $\lambda$ CI 857 , and after infection with wild-type $\lambda$, the phage was induced by exposure to $42^{\circ}$. Restrictionless mutants which had become lysogenic for wild-type $\lambda$ were protected against induction, while the restricting bacteria were lysed. The results of five separate experiments, showing the proportion of nonrestricting mutants found after enrichment and the phenotypes of the mutants, are presented in Table 2. The e.o.p. of $\lambda . \mathrm{K}$ on $\mathrm{hsI}^{+}$strains is about $\mathrm{Io}^{-4}$, whereas the e.o.p. of $\lambda . \mathrm{K}$ on $\mathrm{hsII}^{+}$strains is only $\mathrm{IO}^{-2}$, and this difference in the restriction of $\lambda . \mathrm{K}$ by the two host specificities may account for the greater yield of restrictionless mutants from the strain carrying R $\mathrm{I}_{24}$. Two phenotypes, $\mathrm{r}^{-} \mathrm{m}^{-}$and $\mathrm{r}^{-} \mathrm{m}^{+}$, were observed, and for hsI, where the total number of mutants scored is higher, these two phenotypes occur in roughly equal proportions. 


\section{The uniqueness of hsI and hsII}

It is clear from the results presented in Tables 3 to 6 that the host specificities hsI and hsII are not identical with any of the host specificities previously described. A strain carrying hsI (or hsII) restricts phage $\lambda$ provided the phage $\lambda$ was not grown on a strain carrying hsI (or hsII). Likewise, phage $\lambda$ grown on a strain of $E$. coli $\mathrm{C}$ or an $\mathrm{r}^{-} \mathrm{m}^{-}$mutant of $E$. coli $\mathrm{K}$ or B carrying hsI (or hsII) is restricted by $E$. coli $\mathrm{K}, \mathrm{B}, \mathrm{I} 5$ and $\mathrm{K}(\mathrm{PI})$.

\section{Table 2. The production of restrictionless mutants of $R$ factor-carried host specificities}

Overnight cultures of $\mathrm{R}^{+}$bacteria were diluted $\mathrm{I} / \mathrm{I} 0$ into fresh Oxoid no. 2 broth, incubated for $\mathrm{I} \frac{1}{2} \mathrm{hr}$, harvested by centrifugation and resuspended in $\mathrm{I} / \mathrm{I}$ o the original volume of M9 buffer containing $0.02 \mathrm{M}$-ethylmethane sulphonate. After $60 \mathrm{~min}$. the cultures were diluted 1/50 with $\mathrm{M}_{9}$ buffer and centrifuged. The pellet was resuspended in half the centrifugation volume, in Oxoid no. 2 broth, and incubated overnight. The cultures were now diluted $\mathrm{I} / \mathrm{I} \mathrm{O}$ in fresh broth, and aerated for $c .2 \mathrm{hr}$, followed by a further $\mathrm{I} / 2$ dilution with Oxoid no. 2 broth containing $\mathrm{M} / \mathrm{I} 00 \mathrm{Mg}^{2+}$. Wild-type phage $\lambda . \mathrm{K}$ was added, and after $30 \mathrm{~min}$. the culture was transferred to $42^{\circ}$ and aerated for $60 \mathrm{~min}$. Appropriate dilutions were made and poured in soft agar overlays on VB supplemented plates, and incubated for $48 \mathrm{hr}$ before picking and testing colonies. Unless otherwise stated, incubation was at $30^{\circ}$.

$\begin{array}{lcrr}\text { R factor } & \begin{array}{c}\text { No. of } \\ \text { colonies tested }\end{array} & \begin{array}{c}\text { No. of } \\ \mathbf{r}^{-} \mathbf{m}^{+}\end{array} & \begin{array}{r}\text { No. of } \\ \mathbf{r}^{-} \mathbf{m}^{-}\end{array} \\ \text {R I24 } & 40 & \text { I3 } & \text { I4 } \\ \text { R I24 } & 50 & 7 & \text { I4 } \\ \text { R I32-SS-I } & 58 & 3 & 5 \\ \text { R 3I3-T-I } & 40 & \text { I } & 0 \\ \text { R 3I3-T-I } & 30 & \text { I } & 0\end{array}$

Table 3. Efficiency of plating of phage $\lambda$ vir grown on Escherichia coli $K, B, C, B R I$ and $C R I$

\begin{tabular}{|c|c|c|c|c|c|c|}
\hline \multirow{2}{*}{$\begin{array}{l}\text { Host } \\
\text { strain* }\end{array}$} & \multicolumn{6}{|c|}{ Phage $\dagger$} \\
\hline & $\lambda \cdot \mathrm{K}$ & $\lambda . \mathrm{KRI}$ & $\lambda . \mathbf{B}$ & $\lambda . \mathrm{BRI}$ & $\lambda . \mathrm{C}$ & $\lambda . \mathrm{cRI}$ \\
\hline $\mathbf{K}$ & $I \cdot O$ & $I \cdot O$ & $5 \times 10^{-4}$ & $\mathrm{I} \times \mathrm{IO}^{-3}$ & $4 \times 10^{-4}$ & $\mathrm{I} \times 1 \mathrm{O}^{-4}$ \\
\hline KR I & $4 \times 10^{-4}$ & I'O & $7 \times 10^{-5}$ & $5 \times 10^{-3}$ & $4 \times 10^{-4}$ & $6 \times 10^{-4}$ \\
\hline KR II & $2 \times 10^{-2}$ & $2 \times 10^{-2}$ & $7 \times 10^{-5}$ & $\mathrm{I} \times 1 \mathrm{IO}^{-4}$ & $2 \times 10^{-4}$ & $1 \times 10^{-4}$ \\
\hline B & $2 \times 10^{-4}$ & $5 \times 10^{-5}$ & I $\cdot 0$ & $I \cdot O$ & $\mathrm{I} \times \mathrm{IO}^{-4}$ & $5 \times 10^{-4}$ \\
\hline BRI & $2 \times 10^{-4}$ & $\mathrm{I} \times 10^{-3}$ & $7 \times 10^{-5}$ & $I \cdot O$ & $1 \times 10^{-5}$ & $2 \times 10^{-4}$ \\
\hline BRII & $7 \times 10^{-4}$ & $6 \times 10^{-3}$ & $2 \times 10^{-3}$ & $6 \times 10^{-3}$ & $1 \times 10^{-2}$ & $7 \times 10^{-4}$ \\
\hline C & $1 \cdot 0$ & I.O & I.O & $\mathrm{I} \cdot \mathrm{O}$ & I $\cdot 0$ & I.O \\
\hline CRI & $5 \times 10^{-6}$ & I.0 & $7 \times 10^{-6}$ & $I \cdot O$ & $2 \times 10^{-5}$ & $I \cdot O$ \\
\hline cR II & $4 \times 10^{-3}$ & $\mathrm{I} \times 1 \mathrm{O}^{-2}$ & $1 \times 10^{-4}$ & $\mathrm{I} \times 1 \mathrm{IO}^{-4}$ & $1 \times 10^{-2}$ & $1 \times 10^{-2}$ \\
\hline
\end{tabular}

${ }^{*} \mathrm{~K}$ is c60o, B is $\mathrm{B} t r p, \mathrm{C}$ is $\mathrm{c} t h r, \mathrm{RI}$ is $\mathrm{R} \mathrm{r} 24, \mathrm{RII}$ is $\mathrm{R} 313-\mathrm{T}-\mathrm{I}$.

$\dagger \lambda . \mathrm{K}$ is phage grown on strain $\mathrm{K} ; \lambda . \mathrm{KRI}$ is phage grown on strain $\mathrm{KRI}$, etc.

\section{Complementation studies}

Complementation was tested by transferring the $\mathrm{R}$ factor, or an $h s$ mutant $\mathbf{R}$ factor, to strains carrying the other host specificities, and examining the restriction and modification properties of the resulting strains, with $\lambda$ vir. Controls showing the e.o.p. of variously modified $\lambda$ vir on $\mathrm{R}^{+}$and $\mathrm{R}^{-}$strains are contained in Tables 3 to 6 , 
the phenotypes of $\mathrm{R}^{+}$strains constructed with hsI and hsII $\mathrm{r}^{-}$mutants are recorded in Tables 7 to 9. Although the wild-type host specificities are always expressed, no complementation of the restrictionless mutants by the wild-type host specificity genes was observed. Restriction was not restored to any hsI or hsII $\mathrm{r}^{-}$mutant in the

Table 4. Efficiency of plating of phage $\lambda$ vir grown on Escherichia coli $K, B, C, K R I I, B R I I$ and $C R I I$

\begin{tabular}{|c|c|c|c|c|c|c|}
\hline \multirow{2}{*}{$\begin{array}{l}\text { Host } \\
\text { strain }\end{array}$} & \multicolumn{6}{|c|}{ Phage } \\
\hline & $\lambda . \mathbf{k}$ & $\lambda . \mathrm{kR} I I$ & $\lambda . \mathbf{B}$ & $\lambda$. BRII & $\lambda . \mathrm{C}$ & $\lambda$. cR II \\
\hline $\mathrm{K}$ & $\mathrm{I} \cdot \mathrm{O}$ & I·O & $5 \times 10^{-4}$ & $5 \times 10^{-4}$ & $4 \times 10^{-4}$ & $I \times 10^{-4}$ \\
\hline KR I & $4 \times 10^{-4}$ & $\mathrm{I} \times \mathrm{IO}^{-3}$ & $7 \times 10^{-5}$ & $5 \times 10^{-5}$ & $4 \times 10^{-4}$ & $2 \times 10^{-5}$ \\
\hline KR II & $2 \times 10^{-2}$ & I.O & $7 \times 10^{-5}$ & $7 \times 10^{-3}$ & $2 \times 10^{-4}$ & $\mathrm{I} \times 10^{-3}$ \\
\hline B & $2 \times 10^{-4}$ & $5 \times 10^{-5}$ & I.O & I. 0 & $\mathrm{I} \times 10^{-4}$ & $2 \times 10^{-4}$ \\
\hline BRI & $2 \times 10^{-4}$ & $7 \times 10^{-5}$ & $7 \times 10^{-5}$ & $7 \times 10^{-4}$ & $1 \times 10^{-5}$ & $\mathrm{I} \times 10^{-4}$ \\
\hline BRII & $7 \times 10^{-4}$ & $1 \times 10^{-2}$ & $2 \times 10^{-3}$ & I. 0 & $1 \times 10^{-2}$ & $7 \times 10^{-2}$ \\
\hline $\mathrm{C}$ & I·O & $I \cdot O$ & I $\cdot 0$ & I.O & I.O & $1 \cdot 0$ \\
\hline CRI & $5 \times 10^{-6}$ & $1 \times 10^{-4}$ & $7 \times 10^{-6}$ & $2 \times 10^{-5}$ & $2 \times 10^{-5}$ & $5 \times 10^{-5}$ \\
\hline CR II & $4 \times 10^{-3}$ & I.O & $1 \times 10^{-4}$ & $\mathrm{I} \cdot \mathrm{O}$ & $1 \times 10^{-2}$ & $I \cdot 0$ \\
\hline
\end{tabular}

$\mathrm{K}$ is $\mathrm{C} 600, \mathrm{~B}$ is $\mathrm{B}$ trp, $\mathrm{C}$ is $\mathrm{C} t h r, \mathrm{RI}$ is $\mathrm{R} \mathrm{I} 24, \mathrm{RII}$ is $\mathrm{R} 3 \mathrm{I}_{3}-\mathrm{T}-\mathrm{I}$.

Table 5. Efficiency of plating of phage $\lambda$ vir grown on Escherichia coli $K, K R I, K R I I$ and $K(P I)$

\begin{tabular}{|c|c|c|c|c|}
\hline \multirow{2}{*}{$\begin{array}{l}\text { Host } \\
\text { strain }\end{array}$} & \multicolumn{4}{|c|}{ Phage } \\
\hline & $\lambda . \mathrm{k}$ & $\lambda . \mathrm{KRI}$ & $\lambda . \mathrm{kRII}$ & $\lambda \cdot \mathrm{K}(\mathrm{P} \mathrm{I})$ \\
\hline $\mathrm{K}$ & I’O & $I \cdot O$ & $I \cdot O$ & $1 \cdot 0$ \\
\hline KR I & $4 \times 10^{-4}$ & $1 \cdot 0$ & $\mathrm{I} \times 10^{-3}$ & $3 \times 10^{-4}$ \\
\hline KRII & $2 \times 10^{-2}$ & $2 \times 10^{-2}$ & $\mathbf{I} \cdot \mathbf{O}$ & $5 \times 10^{-2}$ \\
\hline $\mathrm{K}(\mathrm{P} I)$ & $1 \times 10^{-4}$ & $1 \times 10^{-4}$ & $\mathrm{I} \times 1 \mathrm{I}^{-4}$ & I $O$ \\
\hline
\end{tabular}

$\mathrm{K}$ is $\mathrm{C600}, \mathrm{RI}$ is $\mathrm{R} \mathrm{I}_{24}$, and $\mathrm{RII}$ is $\mathrm{R}_{3} \mathrm{I3}_{3}-\mathrm{T}-\mathrm{I}$.

Table 6. Efficiency of plating of phage $\lambda$ vir grown on Escherichia coli 15 , I5RI, I5RII, C, CRI and $C R I I$

Host

strain

$$
15
$$$$
\text { I } 5 \text { RI }
$$$$
\text { I } 5 \text { R II }
$$$$
\mathrm{C}
$$$$
\text { CRI }
$$

CR II
Phage

\begin{tabular}{|c|c|c|c|c|c|}
\hline$\lambda . \mathrm{I} 5$ & $\lambda . \mathbf{I} 5 \mathbf{R} \mathbf{I}$ & $\lambda .15 \mathrm{RII}$ & $\lambda . \mathbf{C}$ & $\lambda . \mathrm{CRI}$ & $\lambda . \mathrm{CRII}$ \\
\hline I $\cdot 0$ & $\mathbf{I} \cdot 0$ & I.O & $3 \times 10^{-2}$ & $5 \times 10^{-2}$ & $I \times 10^{-2}$ \\
\hline$I \times 10^{-2}$ & $I \cdot 0$ & $1 \times 10^{-2}$ & $\mathrm{I} \times 10^{-3}$ & $1 \times 10^{-2}$ & $I \times 10^{-4}$ \\
\hline $\mathrm{I} \times 1 \mathrm{IO}^{-2}$ & $I \times 10^{-2}$ & $I \cdot O$ & $\mathrm{I} \times 1 \mathrm{IO}^{-2}$ & $\mathrm{I} \times 1 \mathrm{IO}^{-2}$ & $\mathrm{I} \times 10^{-2}$ \\
\hline I 0 & $\mathbf{I} \cdot 0$ & I.O & I.O & $1 \cdot 0$ & $I \cdot O$ \\
\hline$I \times 10^{-3}$ & $\mathrm{I} \cdot \mathrm{O}$ & $1 \times 10^{-5}$ & $5 \times 10^{-4}$ & $I \cdot O$ & $1 \times 10^{-4}$ \\
\hline $1 \times 10^{-2}$ & $I \times 10^{-2}$ & I.O & $1 \times 10^{-2}$ & $1 \times 10^{-2}$ & $I \cdot O$ \\
\hline
\end{tabular}

RI is R I24, RII is R $313-T-I$.

presence of intact $\mathrm{K}$, B or P I host specificities (Table 7), or to hsI mutants in the presence of intact hsII (Table 9), or to $\mathrm{r}^{-}$mutants of $\mathrm{K}$ and $\mathrm{B}$ by intact hsI and hsII (Table 8). Nor is modification restored to any $\mathbf{r}^{-} \mathrm{m}^{-}$mutants, although the $\mathrm{m}^{+}$phenotype of $r^{-} m^{+}$mutants is expressed (Tables 7 to 9 ). 
Table 7. Phenotypes displayed by $R^{+}$strains carrying restrictionless mutants of $R I 24, R_{313}-T-I$, and $R I_{32}$

\begin{tabular}{|c|c|c|c|c|}
\hline \multirow[b]{2}{*}{$\mathbf{R}$ factor } & \multirow{2}{*}{$\begin{array}{c}\text { Host- } \\
\text { specificity } \\
\text { phenotype } \\
\text { of } R \\
\text { factor }\end{array}$} & \multicolumn{3}{|c|}{$\begin{array}{l}\text { Host specificity phenotype of the } R^{+} \text {strain } \\
\text { when the host strain is }\end{array}$} \\
\hline & & $\mathrm{K}^{*}$ & B & $\mathrm{K}(\mathrm{PI})$ \\
\hline $\mathrm{R}$ I24 & $\mathrm{r}_{\mathbf{I}}^{+} \mathrm{m}_{\mathbf{I}}^{+}$ & $\mathrm{r}_{\mathrm{I}}^{+} \mathrm{m}_{\mathrm{I}}^{+} \mathrm{r}_{\mathrm{K}}^{+} \mathrm{m}_{\mathrm{K}}^{+}$ & $\mathrm{r}_{\mathrm{I}}^{+} \mathrm{m}_{\mathrm{I}}^{+} \mathrm{r}_{\mathrm{B}}^{+} \mathrm{m}_{\mathrm{B}}^{+}$ & $\mathrm{r}_{\mathrm{I}}^{+} \mathrm{m}_{\mathrm{I}}^{+} \mathrm{r}_{\mathrm{K}}^{+} \mathrm{m}_{\mathrm{K}}^{+} \mathrm{r}_{\mathrm{P1}}^{+} \mathrm{m}_{\mathrm{PI}}^{+}$ \\
\hline R I24-I & $\mathrm{r}_{\mathrm{I}}^{-} \mathrm{m}_{\mathrm{I}}^{+}$ & $\mathrm{r}_{\mathrm{I}}^{-} \mathrm{m}_{\mathrm{I}}^{+} \mathrm{r}_{\mathrm{K}}^{+} \mathrm{m}_{\mathrm{K}}^{+}$ & $\mathrm{r}_{\mathrm{I}}^{-} \mathrm{m}_{\mathrm{I}}^{+} \mathrm{r}_{\mathrm{B}}^{+} \mathrm{m}_{\mathrm{B}}^{+}$ & $\mathrm{r}_{\mathrm{I}}^{-} \mathrm{m}_{\mathrm{I}}^{+} \mathrm{r}_{\mathrm{K}}^{+} \mathrm{m}_{\mathrm{K}}^{+} \mathrm{r}_{\mathrm{P} \mathbf{1}}^{+} \mathrm{m}_{\mathrm{P} \mathrm{I}}^{+}$ \\
\hline $\mathrm{R} I 24^{-2}$ & $\mathrm{r}_{\mathrm{I}}^{-} \mathrm{m}_{\mathrm{I}}^{-}$ & $\mathrm{r}_{\mathrm{I}}^{-} \mathrm{m}_{\mathrm{I}}^{-} \mathrm{r}_{\mathrm{K}}^{+} \mathrm{m}_{\mathrm{K}}^{+}$ & $\mathrm{r}_{\mathrm{I}}^{-} \mathrm{m}_{\mathbf{I}}^{-} \mathrm{r}_{\mathbf{B}}^{+} \mathrm{m}_{\mathbf{B}}^{+}$ & $\mathrm{r}_{\mathrm{I}}^{-} \mathrm{m}_{\mathrm{I}}^{-} \mathrm{r}_{\mathbf{K}}^{+} \mathrm{m}_{\mathrm{K}}^{+} \mathrm{r}_{\mathrm{P} \mathbf{1}}^{+} \mathrm{m}_{\mathrm{P} 1}^{+}$ \\
\hline$R_{1}$ 24-3 & $\mathrm{r}_{\mathrm{I}}^{-} \mathrm{m}_{\mathrm{I}}^{+}$ & $\mathrm{r}_{\mathrm{I}}^{-} \mathrm{m}_{\mathrm{I}}^{+} \mathrm{r}_{\mathrm{K}}^{+} \mathrm{m}_{\mathrm{K}}^{+}$ & $\mathrm{r}_{\mathrm{I}}^{-} \mathrm{m}_{\mathrm{I}}^{+} \mathrm{r}_{\mathrm{B}}^{+} \mathrm{m}_{\mathrm{B}}^{+}$ & $\mathrm{r}_{\mathrm{I}}^{-} \mathrm{m}_{\mathrm{I}}^{+} \mathrm{r}_{\mathbf{K}}^{+} \mathrm{m}_{\mathrm{K}}^{+} \mathrm{r}_{\mathrm{P} \mathbf{1}}^{+} \mathrm{m}_{\mathbf{P} \mathbf{1}}^{+}$ \\
\hline $\mathrm{R}_{3}$ I3-T-I & $\mathrm{r}_{I I}^{+} \mathrm{m}_{I I}^{+}$ & $\mathrm{r}_{\mathrm{II}}^{+} \mathrm{m}_{\mathrm{JJ}}^{+} \mathrm{r}_{\mathrm{K}}^{+} \mathrm{m}_{\mathrm{K}}^{+}$ & $\mathrm{r}_{I I}^{+} \mathrm{m}_{I I}^{+} \mathrm{r}_{\mathrm{B}}^{+} \mathrm{m}_{\mathrm{B}}^{+}$ & $\mathrm{r}_{\mathrm{II}}^{+} \mathrm{m}_{\mathrm{II}}^{+} \mathrm{r}_{\mathrm{K}}^{+} \mathrm{m}_{\mathrm{K}}^{+} \mathrm{r}_{\mathrm{PI}}^{+} \mathrm{m}_{\mathrm{PI}}^{+}$ \\
\hline R 3I 3-I & $\mathrm{r}_{\mathrm{II}}^{-} \mathrm{m}_{\mathrm{II}}^{+}$ & $\mathrm{r}_{\mathrm{II}}^{-} \mathrm{m}_{\mathrm{II}}^{+} \mathrm{r}_{\mathrm{K}}^{+} \mathrm{m}_{\mathrm{K}}^{+}$ & $\mathrm{r}_{\mathrm{II}}^{-} \mathrm{m}_{\mathrm{II}}^{+} \mathrm{r}_{\mathbf{B}}^{+} \mathrm{m}_{\mathbf{B}}^{+}$ & $\mathrm{r}_{\mathrm{II}}^{-} \mathrm{m}_{\mathrm{II}}^{+} \mathrm{r}_{\mathrm{K}}^{+} \mathrm{m}_{\mathrm{K}}^{+} \mathrm{r}_{\mathrm{P} \mathbf{1}}^{+} \mathrm{m}_{\mathrm{P} 1}^{+}$ \\
\hline $\mathrm{R}_{313-2}$ & $\mathrm{r}_{\mathrm{II}}^{-} \mathrm{m}_{\mathrm{II}}^{+}$ & $\mathrm{r}_{\mathrm{II}}^{-} \mathrm{m}_{\mathrm{II}}^{+} \mathrm{r}_{\mathrm{K}}^{+} \mathrm{m}_{\mathrm{K}}^{+}$ & $\mathrm{r}_{\mathrm{II}}^{-} \mathrm{m}_{\mathrm{II}}^{+} \mathrm{r}_{\mathrm{B}}^{+} \mathrm{m}_{\mathrm{B}}^{+}$ & $\mathrm{r}_{\mathrm{II}} \mathrm{m}_{\mathrm{II}}^{+} \mathrm{r}_{\mathbf{K}}^{+} \mathrm{m}_{\mathrm{K}}^{+} \mathrm{r}_{\mathrm{PI}}^{+} \mathrm{m}_{\mathrm{PI}}^{+}$ \\
\hline R3I3-3 & $\mathrm{r}_{\mathrm{II}}^{-} \mathrm{m}_{\mathrm{II}}^{+}$ & $\mathrm{r}_{\mathrm{II}}^{-} \mathrm{m}_{\mathrm{II}}^{+} \mathrm{r}_{\mathrm{K}}^{+} \mathrm{m}_{\mathrm{K}}^{+}$ & $\mathrm{r}_{\mathrm{II}}^{-} \mathrm{m}_{I I}^{+} \mathrm{r}_{\mathrm{B}}^{+} \mathrm{m}_{\mathrm{B}}^{+}$ & $\mathrm{r}_{\mathrm{II}}^{-} \mathrm{m}_{\mathrm{II}}^{+} \mathrm{r}_{\mathrm{K}}^{+} \mathrm{m}_{\mathrm{K}}^{+} \mathrm{r}_{\mathrm{P} 1}^{+} \mathrm{m}_{\mathrm{P} 1}^{+}$ \\
\hline $\mathrm{R} I 32-\mathrm{I}$ & $\mathrm{r}_{\mathbf{I I}^{-}}^{-} \mathrm{m}_{\mathrm{II}}^{+}$ & $\mathrm{r}_{\mathrm{II}}^{-} \mathrm{m}_{\mathrm{II}}^{+} \mathrm{r}_{\mathrm{K}}^{+} \mathrm{m}_{\mathrm{K}}^{+}$ & $\mathrm{r}_{\mathrm{II}}^{-} \mathrm{m}_{\mathrm{II}}^{+} \mathrm{r}_{\mathbf{B}}^{+} \mathrm{m}_{\mathbf{B}}^{+}$ & $\mathrm{r}_{\mathrm{II}}^{-} \mathrm{m}_{+}^{\mathrm{II}} \mathrm{r}_{\mathrm{K}}^{+} \mathrm{m}_{\mathrm{K}}^{+} \mathrm{r}_{\mathrm{P} 1}^{+} \mathrm{m}_{\mathrm{P} 1}^{+}$ \\
\hline $\mathrm{R}_{132-2}$ & $\mathrm{r}_{\mathrm{II}}^{-} \mathrm{m}_{\overline{I I}}$ & $\mathrm{r}_{\mathrm{II}}^{-} \mathrm{m}_{\mathrm{II}}^{-} \mathrm{r}_{\mathrm{K}}^{+} \mathrm{m}_{\mathrm{K}}^{+}$ & $\mathrm{r}_{\mathrm{II}}^{-} \mathrm{m}_{\mathrm{II}}^{-} \mathrm{r}_{\mathrm{B}}^{+} \mathrm{m}_{\mathrm{B}}^{+}$ & $\mathrm{r}_{\mathrm{II}}^{-} \mathrm{m}_{-}^{\mathrm{II}} \mathrm{r}_{\mathrm{K}}^{+} \mathrm{m}_{\mathrm{K}}^{+} \mathrm{r}_{\mathrm{P1}}^{+} \mathrm{m}_{\mathrm{P} 1}^{+}$ \\
\hline R I99-I & $\mathrm{r}_{\mathrm{II}}^{-} \mathrm{m}_{\mathrm{II}}^{+}$ & $\mathrm{r}_{\mathrm{II}}^{-} \mathrm{m}_{\mathrm{II}}^{+} \mathrm{r}_{\mathrm{K}}^{+} \mathrm{m}_{\mathrm{K}}^{+}$ & $\mathrm{r}_{\mathrm{II}}^{-} \mathrm{m}_{\mathrm{II}}^{+} \mathrm{r}_{\mathrm{B}}^{+} \mathrm{m}_{\mathrm{B}}^{+}$ & $\mathrm{r}_{\mathrm{II}}^{-} \mathrm{m}_{\mathrm{II}}^{+} \mathrm{r}_{\mathrm{K}}^{+} \mathrm{m}_{\mathrm{K}}^{+} \mathrm{r}_{\mathbf{P} \mathbf{1}}^{+} \mathrm{m}_{\mathbf{P} \mathbf{1}}^{+}$ \\
\hline
\end{tabular}

$*_{\mathrm{K}}$ strains used were $\mathrm{c} 600$ and $\mathrm{J} 5-3$.

The restriction phenotype was determined by measuring the efficiency of plating of variously modified phage $\lambda$ vir on the test strain, and the modification phenotype was determined by measuring the efficiency of plating on standard strains of the phage $\lambda$ vir grown on the test strain.

Table 8. Phenotypes displayed by restrictionless mutants of Escherichia coli $K$ and $B$ carrying $R_{124}$ or $R_{313-T-I}$

\begin{tabular}{|c|c|c|c|}
\hline \multirow[b]{2}{*}{ Strain } & \multirow{2}{*}{$\begin{array}{c}\text { Phenotype } \\
\text { of } \mathrm{R}^{-} \\
\text {strain }\end{array}$} & \multicolumn{2}{|c|}{ Phenotype of $\mathrm{R}^{+}$carrying } \\
\hline & & $\mathrm{R}_{\mathrm{I} 24}\left(\mathrm{r}_{\mathrm{I}}^{+} \mathrm{m}_{\mathrm{I}}^{+}\right)$ & $\mathrm{R}_{3} \mathrm{I} 3-\mathrm{T}-\mathrm{I}\left(\mathrm{r}_{\mathrm{II}}^{+} \mathrm{m}_{\mathrm{II}}^{+}\right)$ \\
\hline $\mathrm{c} 600$ & $\mathrm{r}_{\mathrm{K}}^{+} \mathrm{m}_{\mathrm{K}}^{+}$ & $\mathrm{r}_{\mathrm{K}}^{+} \mathrm{m}_{\mathrm{K}}^{+} \mathrm{r}_{\mathrm{I}}^{+} \mathrm{m}_{\mathrm{I}}^{+}$ & $\mathrm{r}_{\mathrm{K}}^{+} \mathrm{m}_{\mathrm{K}}^{+} \mathrm{r}_{\mathrm{II}}^{+} \mathrm{m}_{\mathrm{II}}^{+}$ \\
\hline $7 \mathrm{~K}$ & $\mathrm{r}_{\mathrm{K}}^{-} \mathrm{m}_{\mathrm{K}}^{+}$ & $\mathrm{r}_{\mathrm{K}}^{-} \mathrm{m}_{\mathrm{K}}^{+} \mathrm{r}_{\mathrm{I}}^{+} \mathrm{m}_{\mathrm{I}}^{+}$ & $\mathrm{r}_{\mathrm{K}}^{-} \mathrm{m}_{\mathrm{K}}^{+} \mathrm{r}_{\mathrm{II}}^{+} \mathrm{m}_{\mathrm{II}}^{+}$ \\
\hline $4 \mathrm{~K}$ & $\mathrm{r}_{\mathrm{K}}^{-} \mathrm{m}_{\overline{\mathrm{K}}}^{\overline{\mathrm{i}}}$ & $\mathrm{r}_{\mathbf{K}}^{-} \mathrm{m}_{\mathrm{K}} \mathrm{r}_{\mathrm{I}}^{+} \mathrm{m}_{\mathrm{I}}^{+}$ & $\mathrm{r}_{\overline{\mathrm{K}}}^{-} \mathrm{m}_{\overline{\mathrm{K}}}^{-} \mathrm{r}_{\mathrm{II}}^{+} \mathrm{m}_{\mathrm{II}}^{+}$ \\
\hline B & $\mathrm{r}_{\mathrm{B}}^{+} \mathrm{m}_{\mathrm{B}}^{+}$ & $\mathrm{r}_{\mathrm{B}}^{+} \mathrm{m}_{\mathrm{B}}^{+} \mathrm{r}_{\mathrm{I}}^{+} \mathrm{m}_{\mathrm{I}}^{+}$ & $\mathrm{r}_{\mathrm{B}}^{+} \mathrm{m}_{\mathrm{B}}^{+} \mathrm{r}_{\mathrm{II}}^{+} \mathrm{m}_{\mathrm{II}}^{+}$ \\
\hline B 6 & $r_{\mathcal{B}}^{-} m_{B}^{+}$ & $\mathrm{r}_{\mathrm{B}}^{-} \mathrm{m}_{\mathrm{B}}^{+} \mathrm{r}_{\mathrm{I}}^{+} \mathrm{m}_{\mathrm{I}}^{+}$ & $\mathrm{r}_{\mathbf{B}}^{-} \mathrm{m}_{\mathrm{B}}^{+} \mathrm{r}_{\mathrm{II}}^{+} \mathrm{m}_{\mathrm{II}}^{+}$ \\
\hline B 8 & $\mathrm{r}_{\mathbf{B}}^{-} \mathrm{m}_{\mathrm{B}}^{-}$ & $\mathrm{r}_{\mathrm{B}}^{-} \mathrm{m}_{\mathrm{B}}^{-} \mathrm{r}_{\mathrm{I}}^{+} \mathrm{m}_{\mathrm{I}}^{+}$ & $\mathrm{r}_{\mathbf{B}}^{-} \mathrm{m}_{\mathbf{B}}^{-} \mathrm{r}_{\mathrm{II}}^{+} \mathrm{m}_{\mathbf{I I}}^{+}$ \\
\hline
\end{tabular}

Table 9. Phenotypes of C600 RI32 strains also carrying $R I 24$ or a restrictionless mutant or $R 124$

\begin{tabular}{|c|c|c|c|}
\hline & \multicolumn{3}{|c|}{ Host-specificity phenotype } \\
\hline C600 RI32 RI24 & $\mathrm{r}_{\mathrm{K}}^{+} \mathrm{m}_{\mathrm{K}}^{+}$ & $\mathrm{r}_{\mathrm{II}}^{+} \mathrm{m}_{\mathrm{II}}^{+}$ & $\mathrm{r}_{\mathrm{I}}^{+} \mathrm{m}_{\mathrm{I}}^{+}$ \\
\hline c600 RI32 RI24-I & $\mathrm{r}_{\mathrm{K}}^{+} \mathrm{m}_{\mathrm{K}}^{+}$ & $\mathrm{r}_{\mathrm{II}}^{+} \mathrm{m}_{\mathrm{II}}^{+}$ & $r_{I}^{-} m_{I}^{+}$ \\
\hline C600 R I32 R I24-2 & $\mathrm{r}_{\mathbf{K}}^{+} \mathrm{m}_{\mathbf{K}}^{+}$ & $\mathrm{r}_{\mathrm{II}}^{+} \mathrm{m}_{\Pi \mathrm{I}}^{+}$ & $r_{I}^{-} m_{I}^{-}$ \\
\hline $\mathrm{C} 600 \mathrm{R}$ I32 R I 24-3 & $\mathrm{r}_{\mathbf{K}}^{+} \mathrm{m}_{\mathrm{K}}^{+}$ & $\mathrm{r}_{\mathrm{II}}^{+} \mathrm{m}_{\mathrm{II}}^{+}$ & $\mathrm{r}_{\mathrm{I}}^{-} \mathrm{m}_{\mathrm{I}}^{+}$ \\
\hline
\end{tabular}

\section{DISCUSSION}

The absence of efficient quantitative techniques for selecting different restriction or modification phenotypes seriously limits genetic fine structure analysis of host specificities. Although crude mapping and establishment of the allelic relationships 
of the determinants for the host-specificity types $\mathrm{K}$, B and $\mathrm{I}_{5}$ has been established by bacterial conjugation and cotransduction with phage $\mathrm{P}_{\mathrm{I}}$, study of the restrictionless mutants gave the most information concerning the number and function of the genes involved. Complementation studies in partial diploids proved that a minimum of three genes was necessary for the expression of restriction and modification. Provisionally, the following nomenclature for these genes is used: hsm is a gene specifying a product essential for modification, $h s r$ is a gene specifying a product essential for restriction, and $h s s$ is a gene specifying a product essential for both restriction and modification (Arber \& Linn, 1969).

The host-specificity types hsI and hsII are carried on R factors, whose genomes are relatively small compared with the chromosome of Escherichia coli. Mapping of R factors by time of entry studies is not possible since all $\mathrm{R}$ factor-carried determinants appear to enter the recipient at the same time (Meynell et al. 1968). Mapping by phage PI transduction is also excluded, since phage P I normally transduces the entire R factor (Watanabe \& Fukasawa, I96I ; Kondo, Harada \& Mitsuhashi, 1962). Within these limitations, the present analysis of hsI and hsII shows that the phenotypes $\mathrm{r}^{-} \mathrm{m}^{-}$and $\mathrm{r}^{-} \mathrm{m}^{+}$were found for restrictionless mutants of hsI and hsII, the $\mathrm{r}^{-} \mathrm{m}^{-}$ class being observed approximately as frequently as the $\mathrm{r}^{-} \mathrm{m}^{+}$class, and we conclude that both arose as a result of single mutations. By analogy with the $K$ and $B$ host specificities, we anticipate that the hsI and hsII host specificities are controlled by at least three genes, hss, hsr and $h s m$ and that the two phenotypes found, $\mathrm{r}^{-} \mathrm{m}^{-}$and $\mathrm{r}^{-} \mathrm{m}^{+}$ are caused by mutations in hss and $h s r$ respectively.

Further investigation of these host specificities is dependent on complementation studies. Unfortunately, there is incompatibility between $\mathrm{R}$ factors of the same $f$ type, such that two $f^{-}$, or two $f^{+}, \mathrm{R}$ factors will not co-exist stably in the same bacterium (Harada et al. 196I; Mitsuhashi et al. 1962; Watanabe et al. 1964).

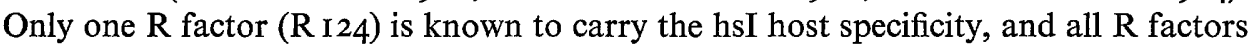
carrying hsII are $f^{-}$(Bannister, I969). Thus it is not possible to test complementation between mutants of hsI, or between mutants of hsII. The only complementation tests possible were between two different host-specificity systems. Complementation does occur between mutants of $\mathrm{K}$ and в (Boyer \& Roulland-Dussoix, I969; Arber \& Linn 1969; Glover, unpublished results) and it was therefore reasonable to test for complementation between hsI and hsII, and between either hsI or hsII and the host specificities $\mathrm{K}$, B and that carried by phage P I. However, in no instance was complementation observed.

The assumption was made that the most suitable test for complementation was the restoration of restriction to an $\mathrm{r}^{-} \mathrm{m}^{+}\left(h s r^{-}\right)$mutant in the presence of the wild-type host specificity of a different type. In addition, $\mathrm{r}^{-} \mathrm{m}^{-}$(presumed $h s s^{-}$) mutants were included, and modification was also scored. This assumption requires that the genetic control of host specificities hsI and hsII is analogous to the genetic control of $\mathrm{K}$ and B host specificities. On the basis of the three-gene model, complementation would involve the association of dissimilar gene products from two host-specificity systems to form a functional enzyme. If the analogous gene products from different host-specificity types were slightly different, association of the gene products might not occur, or the product of association might not function correctly, and complementation would not be observed. A second explanation of the absence of complementation is possible. The apparent similarity of the hsI and hsII host specificities to the $\mathrm{K}$ and B host 
specificities may be only superficial, and at the molecular level there may be fundamental differences.

It has been argued, in relation to host specificity mutants of phage P I, that the absence of complementation with the intact $\mathrm{K}$ and $\mathrm{B}$ host specificities is no indication that complementation could not occur, because such restrictionless mutants of phage $P_{I}$ are normally selected in $\mathrm{K}$ strains. Hence, the phage* ${ }_{\mathrm{z}}$ I mutants are preselected for the inability to complement with the $\mathrm{K}$ host specificity, and, it may be assumed, with the в host specificity also. This criticism is also true of the mutants of hsI and hsII, which have been selected in a $\mathrm{K}$ strain. However, mutants of $\mathrm{K}$ and $\mathrm{B}$ host specificities, known to complement other mutants ( $7 \mathrm{~K}$ and $\mathrm{B} 6$ in Table 8), failed to complement with the intact hsI and hsII host specificities. Hence it may be assumed that the absence of complementation is indicative of an inherent difference between the host specificity systems, rather than to the nature of the mutants.

One of us, D. B., is indebted to the M.R.C. for a Scholarship for training in research methods.

This work formed part of a Ph.D. thesis by D. B. presented to Edinburgh University.

\section{REFERENCES}

APPleyARD, R. K. (1954). Segregation of new lysogenic types during growth of a doubly lysogenic strain derived from Escherichia coli $\mathrm{K} \mathrm{I} 2$. Genetics 39, 440.

ARBER, W. (1968). Host-controlled restriction and modification of bacteriophage. Symposium of the Society for General Microbiology 18, 296.

Arber, W. \& Dussolx, D. (I962). Host specificity of DNA produced by Escherichia coli. I. Host controlled modification of bacteriophage $\lambda$. Journal of Molecular Biology 5, i8.

Arber, W. \& LinN, S. (1969). DNA modification and restriction. Annual Review of Biochemistry $3^{8}, 467$.

BANNISTER, D. (1969). Restriction and modification controlled by resistance transfer factors. Ph.D. thesis, Edinburgh University.

BanNister, D. \& Glover, S. W. (1968). Restriction and modification of bacteriophages by $\mathrm{R}^{+}$ strains of Escherichia coli $\mathrm{K}$ I2. Biochemical and Biophysical Research Communications 30, 735.

BOYER, H. (1964). Genetic control of restriction and modification in Escherichia coli. Journal of Bacteriology 88, 1652 .

Boyer, H. \& Roulland-Dussoix, D. (1969). A complementation analysis of the restriction and modification of DNA in Escherichia coli. Journal of Molecular Biology 4r, 459.

Clowes, R. C. \& Rowley, D. (I954). Some observations on linkage effects in genetic recombination in Escherichia coli $\mathrm{x} \mathbf{1 2}$. Journal of General Microbiology Ir, 250.

Colson, C., Glover, S. W., Symonds, N. \& Stacey, K. A. (1965). The location of the genes for host controlled modification and restriction in Escherichia coli K I2. Genetics 52, I043.

Glover, S. W. \& Colson, C. (1969). Genetics of host controlled restriction and modification in Escherichia coli. Genetical Research $13,227$.

Glover, S. W., Schell, J., Symonds, N. D. \& Stacey, K. A. (1963). The control of host-induced modification by phage Pr. Genetical Research 4, 480 .

Harada, K., Kameda, M., Suzuki, M., Kakinuma, Y. \& Mitsuhashi, S. (I96i). Drug resistance of enteric bacteria. I3. Interference of $\mathrm{R}$ factors in the $\mathrm{R}^{+}$bacterial cell when infected with another R factor. Gunma. Journal of Medical Sciences ro, 201.

Hoekstra, W. P. M. \& De HaAn, P. G. (1965). The location of the restriction locus for $\lambda . \mathrm{K}$ in Escherichia coli $\mathrm{B}$. Mutation Research 2, 204.

JACOB, F. \& Wollman, E. L. (1954). Etude génétique d'un bactériophage tempère d'Escherichia coli: Le système génétique du bactériophage $\lambda$. Annales de l'Institut Pasteur 87, 653 .

Kondo, E., Harada, K. \& Mitsuhashi, S. (1962). Drug resistance of enteric bacteria. I2. Transduction of the transmissible drug resistance factor by bacteriophage P I kc. Japanese Journal of Experimental Medicine 32, 139. 
LEDERBERG, S. (I966). Genetics of host controlled restriction and modification of deoxyribonucleic acid in Escherichia coli. Journal of Bacteriology 31, 1079.

LeDerberg, E. M. \& LeDerberg, J. (I953). Genetic studies of lysogenicity in Escherichia coli. Genetics $\mathbf{3}^{8}, 5 \mathrm{r}$.

LURIA, S. E. (I953). Host induced modification of viruses. Cold Spring Harbour Symposia on Quantitative Biology 18, 237.

Meselson, M. \& YuAN, R. (I968). DNA restriction enzyme from E. coli. Nature 217, il IO.

Meynell, E., Meynell, G. G. \& Datta, N. (1968). Phylogenetic relationships of drug resistance factors and other transmissible plasmids. Bacteriological Reviews 32, 55.

Mitsuhashi, S., Harada, K., Hashimoto, H., Kameda, M. \& Suzuki, M. (I962). Combination of two types of transmissible drug-resistance factors in a host bacterium. Journal of Bacteriology 84, 9.

StACEY, K. A. (1965). Intracellular modification of nucleic acids. British Medical Bulletin 2I, 2 II.

SusSman, R. \& JACOB, F. (1962). Sur un système de répression thermosensible chez le bactériophage $\lambda$ d'Escherichia coli. Compte Rendu Hebdomadaire des Séances de l'Académie des Sciences 254, 1517.

VoGeL, H. J. \& BoNNER, D. M. (1956). Acetylornithinase of Escherichia coli: partial purification and some properties. Journal of Biological Chemistry 218, 97.

WATANABE, T. (1963). Infective heredity of multiple drug resistance in bacteria. Bacteriological Reviews 27, 87.

Watanabe, T. \& FukASAWA, T. (I96I). Episome-mediated transfer of drug resistance in Enterobacteriaceae. III. Transduction of resistance factors. Journal of Bacteriology 82, 202.

Watanabe, T., Nishida, H., Ogata, C., Arai, T. \& Saito, S. (1964). Episome-mediated transfer of drug resistance in Enterobacteriaceae. VII. Two types of naturally occurring $R$ factors. Journal of Bacteriology 88, 716.

WooD, W. B. (1965). Mutations in E. coli affecting the host controlled modification of bacteriophage $\lambda$. Pathologia et Microbiologica 28, 73.

WooD, W. B. (1966). Host specificity of DNA produced by Escherichia coli: Bacterial mutations affecting the restriction and modification of DNA. Journal of Molecular Biology 16, I 18. 